\title{
¿Por qué Chile? Un análisis post crítico sobre los discursos de escala en la movilidad académica internacional
}

\author{
Por que Chile? Uma análise pós-crítica sobre os discursos de escala \\ na mobilidade acadêmica internacional
}

\author{
Why Chile? A post-critical analysis about scalar discourses of \\ international academic mobility
}

CÉSAR Augusto FERRARI MARTINEZ $@$

Universidade Federal de Pelotas, Pelotas, RS, Brasil.

\begin{abstract}
RESUMEN
La movilidad académica internacional es parte de una trama de políticas de internacionalización que producen diferencia entre lo global y lo local, definiendo lo que se consideran buenas prácticas en la pedagogía y en la gestión universitarias. Está basada en imaginarios espaciales fijos acerca de la globalización, de las fronteras, de las naciones y de los movimientos. De la misma manera, fundamentada en prácticas de mercado que utilizan estrategias empresariales para clasificar y jerarquizar, la internacionalización produce lugares de excelencia y sostiene una cartografía epistemológica asimétrica. En este artículo, se identifican en la literatura enunciados lineales de escala tales como interculturalidad, inmersión, campus internacional, entre otros. Nuevas herramientas teóricas son propuestas para complejizar y profundizar tales conceptos, por las cuales se entiende que las trayectorias de los estudiantes interncionales no son estáticas.
\end{abstract}

Palabras clave: Internacionalización. Movilidad académica internacional. Escala. Acercamiento post crítico.

\section{RESUMO}

A mobilidade acadêmica internacional é parte de uma trama de políticas de internacionalização que produzem diferença entre o global e o local, definindo o que se considera como boas práticas na pedagogia e gestão universitárias. Está baseada em imaginários espaciais fixos sobre a globalização, as fronteiras, as nações e os movimentos. Da mesma forma, fundamentada em práticas de mercado que utilizam estratégias corporativas para classificar e hierarquizar, a internacionalização produz lugares de excelência e sustenta uma cartografia epistemológica assimétrica. Neste artigo, se identificam na literatura enunciados lineais de escala, tais como interculturalidade, imersão, campus internacional, entre outros. Novas ferramentas teóricas são propostas para complexificar e aprofundar tais conceitos, pelas quais se entende que as trajetórias dos estudantes não são estáticas.

Palavras-chave: Internacionalização. Mobilidade acadêmica internacional. Escala. Abordagem pós-crítica.

\begin{abstract}
International academic mobility is part of a web of internationalization policies that produce a difference between global and local scales, defining what is considered good practice in terms of pedagogy and university management. It based on fixed spatial imaginaries about globalization, borders, nations and movement. In the same way, corroborated by market practices, which use corporate strategies to classify and hierarchize, internationalization produces places of excellence and supports an asymmetrical epistemological cartography. This article aims to investigate lineal scale enunciations in the literature, such as interculturality, immersion, international campus, among others. New theoretical tools are proposed to complex and deepen such concepts, promoting the understanding of international student's path as non-static.
\end{abstract}

Keywords: Internationalization. International academic mobility. Scale. Post-critical approach. 


\section{¿Por QUÉ ChIle? GATIllando EXPERIENCIAS GEOPOLÍTICAS}

La teorización que direcciono en este texto parte de mi experiencia como investigador novel brasileño de irme a estudiar en otro país. Anclado en una educación superior pautada por discursos de internacionalización y motivado a investigar movilidad académica internacional, pensé que estudiar en el extranjero podría ser una manera de complejizar mi trayectoria profesional y personal. La decisión de irme a estudiar en Santiago de Chile fue producto de la convergencia de múltiples discursos y experiencias. Por un lado, soy producto del discurso normativo de la excelencia y de la calidad, pues no es menor el hecho de que la Facultad de Educación de la Pontificia Universidad Católica de Chile, institución que me acogió en mis estudios doctorales, goce de excelente ubicación en rankings globales dentro de su área. Por otro lado, también adopté el contra-discurso de realizar un doctorado en Latinoamérica y no en el mundo anglosajón, habiendo imaginado que desde ese espacio yo podría refutar a los flujos intuitivos y coloniales que yo mismo criticaba. Otros elementos se fueron incorporando a mi interés por moverme, tales como acceso a becas, disposición para aprender español, pretensiones de vivir en Santiago, estar cerca de mi familia en Brasil. Para mí, estar en Chile era, por lo tanto, un movimiento muy intuitivo, a la vez que yo llevaba en mente un largo listado de argumentos que justificaban la decisión.

A pesar de mi convicción, en mi primera semana de clases en la Universidad, asistí a la ponencia de una reconocida profesora brasileña. Al término, fui a hablar con ella para hacerle algunas preguntas $\mathrm{y}$, al contarle que estaba haciendo el doctorado en Chile, me miró con gran sorpresa y me preguntó: Pero ¿por qué Chile? Me sentí afectado, puesto en duda, pero busqué relevar a su reacción y seguir con mis actividades académicas en las siguientes semanas. No obstante, aquella pregunta que me interpeló la primera vez siguió pronunciándose en la voz de académicos y académicas de tal manera que, sintiéndola reverberar, pasé a creer que estaba en un movimiento contraintuitivo, que había salido de la grilla. Cuando retomaba mi tema de investigación, tenía en claro que la neoliberalización de la academia era fuertemente afectada por los estándares de accountability. Sin embargo, percibí que, aunque estuviese estudiando en una universidad posicionada como una de las mejores del mundo por esos mismos estándares, estaba designado a un espacio periférico. Yo vivía la condición de ser afectado por la geopolítica, tenía mi cuerpo como un resultado de una geografía normativa. ¿Por qué no Chile?

Mi interés en ese tema se despliega de asumir que el espacio epistemológico se produce en los movimientos de estudiantes. Comprendo, conforme argumento aquí, que la cartografía de la ciencia está conformada en discursos que atribuyen valores, que homogenizan y que jerarquizan esos espacios. También entiendo que el conocimiento puede ser un vehículo de posibilidad, convirtiéndose en un evento que desestabiliza a los territorios. En este artículo, me dedico a complejizar algunos de los movimientos de estabilización y desestabilización de las cartografías epistemológicas teniendo en cuenta las trayectorias vividas por estudiantes y cómo sus conocimientos se van produciendo a partir de cuerpos transformantes y reorientados por la movilidad. Parto de una narrativa personal y de cómo este problema de la geopolítica académica es tratado por la literatura internacional. Busco, así, entender la geopolítica corporizada en una movilidad académica geográficamente normativa. Lo hago a través del mapeo de los discursos de escala, observando como el poder circula entre las políticas de internacionalización y atribuye ciertos valores a los espacios.

\section{LA MOVILIDAD ACADÉMICA COMO FENÓMENO EN LA EDUCACIÓN SUPERIOR}

La Movilidad Académica Internacional (MAI en adelante) no es un fenómeno nuevo, pero su aumento ha sido notable en las últimas décadas (DOLBY, 2008). Después de haber crecido más de $100 \%$ en apenas diez años (United Nations Educational Scientific and Cultural Organization [UNESCO], 2016), parte de los expertos y de las expertas en educación internacional pasaron a percibir los movimientos como más que una simples decisión, sino como un evento socio-político. En la literatura, se pasó a consensuar que el discurso vigente para producir MAI era el mismo discurso de la globalización (ver por ejemplo FINDLAY et al., 2012; RIZVI, 2011; SHIELDS, 2014). Sin embargo, las conceptualizaciones de lo que es globalización son muy distintas entre las diferentes tradiciones académicas.

La MAI no es un fenómeno aislado, sino que es parte de una compleja trama de políticas de internacionalización. Es importante mencionar que la movilidad atribuye sentido a muchas de esas políticas, que incluyen becas, programas de idiomas, adecuaciones curriculares, estandarizaciones internacionales, publicaciones internacionales, entre tantas otras (MATUS, 2016). Si antes esas prácticas eran resultado de movimientos individuales por parte de grupos de elite nacionales, en los últimos años ellas vienen convirtiéndose en políticas institucionales. En otras palabras, hay una preocupación por parte de gobiernos, agencias y universidades para consolidar la internacionalización y la MAI es uno de los medios más eficientes para alcanzar ese objetivo. 
En los años recientes, las naciones sudamericanas han invertido cifras significativas para promocionar la MAI a través de políticas de estado. El programa Ciência sem Fronteiras de Brasil, por medio de un consorcio público y privado, generó más de 90 mil becas en el extranjero en poco más de 5 años. El programa de becas de la Organización de los Estados Americanos se apoya en las inversiones públicas de los países para estimular la movilidad interna entre ciudadanos de naciones latinoamericanas. Desde 2014, la Comisión Nacional de Investigación Científica y Tecnológica de Chile viene incorporando a los extranjeros como candidatos a postulantes a las becas nacionales de postgrado. En conjunto, las medidas mencionadas insertan el tema de la movilidad en los cotidianos de las universidades sudamericanas y en la pauta pública de discusiones.

Si entendemos las políticas de internacionalización como estándares que evalúan la calidad de las universidades, tendemos a comprender que ellas afectan no solo a los estudiantes en movilidad, pero a imaginarios y a los criterios de lo que se consideran buenas universidades, buenas prácticas, buenos y buenas estudiantes (MATUS; TALBURT, 2015). La MAI, en ese caso, es, al mismo tiempo, una manera de consolidar políticas de internacionalización y una práctica de distinción entre lo internacional y lo local. En resumen, una política que produce diferencia y que les designa lugares a estudiantes y a instituciones en la jerarquía del conocimiento. Además, es una política que juega con discursos de escala y de consumo de esas escalas. En suma, la MAI es la globalización en su dimensión académica.

\section{CóMO SE HA ESTUDIADO MOVILIDAD ACADÉMICA INTERNACIONAL}

La revisión de la literatura de los últimos años demuestra que hay tres grandes perspectivas paradigmáticas acerca de la MAI. En un primer acercamiento, se percibe la globalización como un fenómeno consecuente y natural se asume la MAI como una buena práctica para actuar en tiempos globales. Por otro lado, se constata una corriente crítica que establece denuncias a como ese proceso global genera inequidad entre países, sujetos e instituciones. Finalmente, un grupo que se mueve por conversaciones postestructurales pone en duda los supuestos normativos por los cuales se viene pensando la MAI.

La MAI, en una perspectiva optimista, toma como natural el proceso de globalización, asumiéndolo como una progresiva integración de los espacios del mundo y como una fragilización de las fronteras. En ese concepto, la MAI es una herramienta por la cual se forman a los y a las estudiantes para un mundo de diversidad cultural (BRODIN, 2010; LO-PHILIP et al., 2015). El rol de las universidades es, asimismo, el de prepararles que califiquen de la mejor manera sus experiencias en el extranjero (BALDASSAR \& MCKENZIE, 2016; DOYLE, GENDALL, MEYER, HOEK, \& TAIT, 2010). A ese grupo de autores, la MAI es un atributo que desarrolla competencias culturales y cognitivas personales y a partir de la exposición a contextos culturales diversos. Por lo anterior, se destacan conceptos clave como interculturalidad (FORSEY, BROOMHALL, \& DAVIS, 2012), ciudadanía global (HIGGINS; BRADY, 2016; WYNVEEN; KYLE; TARRANT, 2012) e inmersión (KILLICK, 2011; SCHARTNER, 2016).

Ante la denuncia de que esa perspectiva optimista de la MAI no incorporaba factores macroestructurales, investigadores e investigadoras de posición crítica agregaron la visión sistémica a los análisis. Esos estudios de macroflujos demuestran que los estudiantes poco eligen, pues están designados por estructuras sociales y políticas (BARNETT et al., 2015; CHANKSELIANI, 2016). Shields (2014) analizó datos sobre los movimientos globales de estudiantes y concluyó que los flujos están cada vez más concentrados, corroborando las conclusiones de Perkins y Neumayer (2014), quienes encontraron enlaces coloniales entre las decisiones de destinación de estudiantes internacionales. Los estudios de Findlay (2011; FINDLAY et al., 2012) indican que la movilidad académica, además de sus atributos pedagógicos, sirve como reproducción de una clase de elite global, pues demuestra que la decisión de estudiar en otro país es una estrategia de reproducción de capital cultural y social. Para la corriente crítica, la globalización es un proceso que afirma fronteras y actúa regulando a los sujetos en el espacio. Esa selectividad, articulada con la demanda por moverse, produce una geopolítica contradictoria y en tensión (KING; RAGHURAM, 2013; TANNOCK, 2013).

Estudios postestructurales aportan para deconstruir a los conceptos fundacionales que operan en estudios de educación internacional. Una de las principales críticas es al respecto de la linealidad entre sujeto y espacio, en una geografía estática. Sidhu y Dall'Alba (2012), por ejemplo, critican el entendimiento dicotómico de la categoría estudiante internacional. Para las investigadoras, el binomio nacional/extranjero (insider/outsider) no es suficiente para comprender el devenir de los estudiantes en el espacio. Cuando se asume esa relación dicotómica en la que la cultura está centrada en la nación, se ignoran todos los procesos y los cambios presentes en la movilidad estudiantil (RIZVI, 2011). Doerr (2014) problematiza algunas de las ideas vigentes en los discursos de MAI, como interculturalidad e inmersión, cuando demuestra 
que los discursos de educación internacional son discursos productores de escala, porque anuncian la cultura como un atributo de quienes vienen de ciertas naciones. $\mathrm{Al}$ imaginar los territorios como receptáculos vacíos, se abre camino para una forma de pensar que inscribe a los sujetos a esos espacios (MATUS, 2016), tratando la diferencia de manera folclórica y/o estigmatizada (DOUGLASS, 2015).

La producción de la literatura reciente ha mostrado que más que un tema curricular, la MAI es un complejo juego geopolítico y que es atravesada por múltiples discursos geográficos normativos. Las universidades aparecen como agentes clave no solo de producción de conocimiento, sino de prácticas de mercado (MATUS, 2016; MATUS; TALBURT, 2009). La adopción de políticas de internalización demanda recursos públicos (DENNIS, 2016), afecta políticas de frontera (WALKER, 2013), cambia dinámicas urbanas (COLLINS, 2014), entre otros tantos fenómenos relacionados. Para comprender la MAI en su profundidad, los sujetos que se mueven para estudiar no pueden ser reducidos a la categoría de estudiantes, ni tampoco ignorados por ella. Se constituyen estudiantes internacionales mientras son atravesados por discursos variados acerca de sus conocimientos, en una trama compleja con otros discursos que los nombran y les atribuyen valor.

\section{LAS UNIVERSIDADES Y LA PRODUCCIÓN DE INTERNACIONALIZACIÓN}

El proceso de internacionalización se caracteriza por un quehacer común entre las universidades alrededor del mundo (COLLINS et al., 2014). Sidhu y Dall'Alba (2012) demuestran que la universidad pasa a utilizar los criterios que evaluaban mercados en la evaluación de sujetos, instituciones y espacios. Se adoptan ciertos estándares en la medición de la productividad y esos estándares resultan en calificaciones a partir de las cuales se compara, se distribuye y se jerarquiza. Por lo tanto, el proceso por el cual se establece la lógica neoliberal en la academia comienza por entender el propio rol de la universidad en las políticas espaciales. La consolidación de regímenes de excelencia es una articulación entre una noción neoliberal de calidad y artefactos discursivos que atribuyen eficacia a los espacios. Los rankings universitarios, por ejemplo, son uno de esos aparatos que refuerzan el rol de la nación asociada a instituciones de excelencia académica (GONG; HUYBERS, 2015).

La educación superior en tiempos de economía del conocimiento (CHIANG, 2012) opera con las mismas estrategias que las corporaciones transnacionales, ubicándose en nodos de los flujos globales de capital (SHIELDS, 2014a) y consolidando las estrategias de la globalización (PHERALI, 2012). La educación transnacional, en ese proyecto, vendría a ser la formación de capital humano necesaria para el desarrollo económico de las empresas transnacionales. De esa manera, hubs de movilidad académica se instalan en países clave para los flujos financieros, sobre todo en la Asia oriental, en naciones como Singapur (COLLINS et al., 2014; WEISS; FORD, 2011), Malasia (ABD AZIZ; ABDULLAH, 2014; WEISS; FORD, 2011) y Hong Kong (GAO, 2014; WATERS; LEUNG, 2016; ZIGURAS; PHAM, 2014). La actuación de esas instituciones adopta el mismo proceso de branding que cualquier operación comercial, acogiendo o comprando nombres de universidades reconocidas para legitimarse y, así, controlar mercados (HEALEY; MICHAEL, 2014). Las investigaciones en ese campo han demostrado que la relación entre esas instituciones no pueden entenderse como cooperación científica, una vez que no son simétricas, pues presentan una relación matriz/ sucursal (HEALEY; MICHAEL, 2014) y reproducen un modelo competitivo y meritocrático (WATERS; LEUNG, 2016).

Ye (2015) destaca que la práctica de transnacionalización es productora de diferencia, una vez que se producen posiciones de privilegios entre estudiantes. Los que pueden ingresar a esos hubs pasan a ser vistos como capaces de acceder a redes globales de excelencia (FURUKAWA; SHIRAKAWA; OKUWADA, 2013). El proceso que Geddie (2015, traducción propia) denomina como "mercantilización en red" sostiene universidades con objetivos corporativos, erosionando los límites entre producción y conocimiento (RIZVI, 2009). En esa dinámica, nuestra vida se ha tornado una herramienta capitalista en la que el producto, en última instancia, somos nosotros y nosotras (MASSUMI, 2015). El movimiento en sí mismo, en su capacidad de conectar, de afectar y de ser afectado, pasa a ser la escala de deseo de la movilidad.

\section{LA INTERNACIONALIZACIÓN Y LA PRODUCCIÓN DE CONOCIMIENTOS}

De acuerdo con Massey (2008), la globalización es una narrativa que se cuenta como si fuera un proceso natural y que hace con que ignoremos que es un proyecto geopolítico. Los mercados instalan un discurso que lleva a creer que las personas y los espacios están globalmente conectados (RIZVI, 2011). Ese "nuevo normal", como lo describen Denzin y Giardino (2017), nos produce una subjetividad que establece a la productividad, a los factores de impacto, a las métricas como la única forma posible de actuar en la academia. Además, nos configura como promotores de nuestra propia necesidad de impactar. Somos afectados de tal manera que nuestros cuerpos son 
las máquinas del deseo de la producción (DELEUZE; GUATTARI, 1997; MASSUMI, 2015). Somos nuestra propia tecnología de gobierno, moviéndonos para los checkpoints requeridos para legitimarnos continuamente (MASSUMI, 2015). En la MAI, ese proceso genera una búsqueda por acceder a espacios de excelencia, un deseo por cartografiar nuestra calidad. No solo las instituciones pasan a ser valoradas, sino también las naciones y las regiones. Cefai (2014, traducción propia) denomina a esas prácticas como "mercantilización del deseo", en lo cual los estudiantes se mueven para consumir escalas. Dos consecuencias primordiales se despliegan de esos discursos: 1) la producción de un régimen de diferencia entre académicos y académicas; 2) la manutención de una cartografía asimétrica entre espacios. Argumento que ambas se relacionan.

En lo que se refiere a la primera, investigaciones han demostrado como la perpetuación de esas prácticas puede generar inequidad en los medios universitarios. La mayoría busca flujos por los que puedan desplazarse a países de habla inglesa (YE, 2015), en un proceso de conectividad selectiva (GEDDIE, 2015). Esta ventaja de dominar la lengua inglesa se transforma en una diferencia que produce privilegios entre los sujetos, conforme lo señalan Sterzuk (2014) y Sibomana (2016). El hecho de, por ejemplo, haber cursado un postgrado en un país de habla inglesa proporciona mejores condiciones para poder publicar en esa lengua, para poder presentar en congresos internacionales, para revisar artículos para revistas extranjeras y para impulsar a tantos otros factores de medición pautados por los estándares internacionales. La consecuencia de ese proceso es una sobrealimentación del mundo anglosajón, pues académicos y académicas de países de habla inglesa y de otros países están produciendo para alimentar un mercado de conocimiento que es fundamentalmente del norte global. Luego, en una relación lineal entre espacios y sujetos, podemos sobrevalorar también los que representan esas epistemologías: blancos, hombres, angloparlantes. La anamorfosis abajo (Imagen 1) representa las áreas de los países proporcionarles a las métricas de la producción académica en ciencias sociales en 2015. Ilustra con precisión el exagero productivo a que se atribuye a ciertas naciones europeas y norteamericanas.

Imagen 1 - Anamorfosis de la producción científica internacional en ciencias sociales (2015)

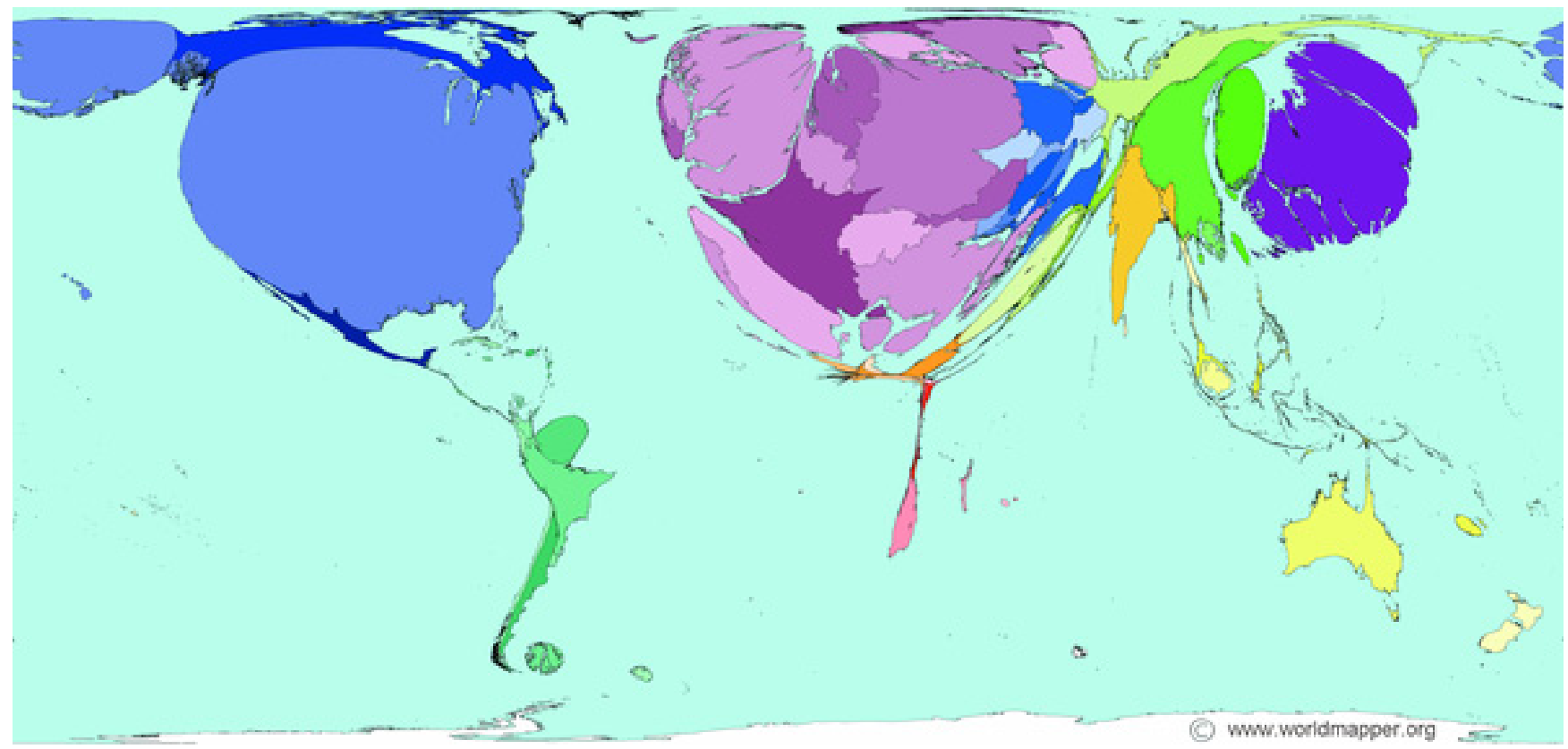

Fuente: Imagen capturada de "It's time to redraw the world's very unequal knowledge map" (8 jul. 2015)1.

\footnotetext{
CZERNIEWICZ, Laura (Comp.). It's time to redraw the world's very unequal knowledge map. The Conversation. [s. I.], 8 jul. 2015. If You Map The World By Scientific Research Output, Things Look Rather Uneven, online. Disponível em: https://theconversation.com/its-time-to-redraw-the-worlds-veryunequal-knowledge-map-44206. Acesso em: 29 jan. 2019.
} 
En otras palabras, las políticas de internacionalización de países anglosajones promueven sus propias naciones en estándares que ellos mismos crearon. Las políticas de internacionalización de países periféricos, como Brasil o Chile, también tienen como objetivo incrementar los estándares de los países de habla inglesa, generando una inequidad evidente en el diseño epistemológico global. La globalización promocionada por los mercados de educación superior disfraza de integración los regímenes de dominación epistémicos, designando geopolíticas asimétricas para los medios académicos. Nuestros cuerpos son afectados insistentemente por discursos de escala sobre ciertas naciones, que estimulan la producción de un sentido de vitalidad (MASSUMI, 2015, p. 23) que nos impele a desearlas.

\section{LOS DISCURSOS DE ESCALA}

La escala es un concepto polisémico que operó por mucho tiempo invisibilisado en las ciencias sociales, incluso en la propia Geografía. Su definición más amplia es la dimensión espacial de un evento. No obstante, en los años noventa (SMITH, 1992) y en los años dos mil (BRENNER, 2001; MARSTON, 2000; MARSTON; SMITH, 2001; MARTINEZ, 2018a) el concepto fue desnaturalizado y descentrado de la cartografía positivista y pasó a ser una herramienta importante en la comprensión de la producción política espacial. La escala pasó a ser entendida como un discurso que establecía a los espacios, lo que la llevo a ser analizada en lo que se refiere a su carácter gubernamental, performativo y epistemológico. La escala como está presentada aquí, en el marco de una cartografía postrepresentacional (AZÓCAR \& BUCHROITHNER, 2013), no es por lo tanto la dimensión métrica de un hecho (área de ocurrencia), sino cómo se sostienen los discursos espaciales acerca de ese mismo hecho. Eso requiere entenderla no como una estructura rígida y organizativa del espacio (nested scales) y sí como una instancia enunciativa, reiterando performativamente las relaciones de poder entre los lugares.

Una de las contribuciones más importantes fue desarrollada por Brown, que en conjunto con Knopp (2006, 2011) y Legg (2013) argumentan de que la escala actúa como estrategia de gubernamentalidad. De acuerdo con ellos, la representación del mapa con sus límites definidos refuerza la idea de que quienes habitan esos espacios comparten algo. Además, reitera nociones de espacio fijo con límites bien establecidos, el espacio como una unidad homogénea y estable. Collinge (2005), basado en el trabajo de Swyngedouw, resalta que la escala viene siendo ocupada por los trabajos con enfoque espacial: produciendo unidades espaciales análogas para poder jerarquizar y comparar, encubriendo las diferencias entre objetos fijos (edificios, por ejemplo) y móviles (familias), asociando área y pertenencia como atributos de la misma escala y confundiendo la unidad de área con el espacio de acción institucional. Con esos análisis, lo defendido por Collinge y otros autores es de que la escala no es solo una herramienta analítica, sino que también un potente enunciado que produce una fusión entre área, territorio y lugar en un discurso totalizante. Las escalas son estrategias de poder que producen espacio discursivamente, instancias enunciativas que producen geografías normativas.

Al asumir que estudiantes son atravesados por esas escalas de excelencia en movilidad académica, planteo considerar sus prácticas discursivas como escalas performativizadas. La identidad espacial es un poder recursivo que sitúa a los sujetos geográficamente en condiciones a la posibilidad (MARTINEZ, 2012; NELSON, 1999). La performatividad de los espacios es, por lo tanto, la producción duradera y modulada de discursos de escala, reinstalando cartografías de gobierno. La nación, por ejemplo, es resultado de esos esfuerzos continuos por afirmar la estabilidad de ciertos espacios, lo que permite producir relaciones de poder entre ellos, además de clasificarlos y jerarquizarlos (COLLINGE, 2005; SWYNGEDOUW, 2004). El discurso nacional es un enunciado, una metarranativa (LYOTARD, 1984) que encuentra en la movilidad académica una estrategia reproductiva. Las fronteras son resultados de esas cartografías escalares, pues los estudiantes están cargados de identidades atribuidas (DOUGLASS, 2015), imponiéndoles geopolíticas cotidianas. Desde la geografía de la educación, pongo atención en que acciones emergen para sostener o desestabilizar esos continuos normativos entre las trayectorias de los estudiantes en movilidad. Además de ello, si la escala es un constructo por el cual se discursa geopolíticamente los órdenes espaciales, ella tiene una implicación en cómo producimos conocimiento. Es una forma de mirarlo, pensarlo, ordenarlo (KAISER; NIKIFOROVA, 2008). En considerar la escala una epistemología, la geografía de la educación abre un importante canal para aportar deconstructivamente a esos regímenes epistemológicos. Las escalas son discursos por los cuales el poder se ejerce sobre/por los espacios.

\section{LOS DISCURSOS DE ESCALA QUE SE SOSTIENEN POR LAS POLÍTICAS DE INTERNACIONALIZACIÓN}

Hay tres grandes discursos de escala que sobresalen en la literatura y que vienen siendo objeto de crítica y de disputa por los campos, estableciendo en prácticas de movilidad lo que yo denomino de geografías normativas. Por un lado, está la noción de interculturalidad, que se 
entiende como el conjunto de prácticas y de conocimientos en común que son compartidos por grupos nacionales y que pueden ser intercambiados como una mercancía en las trayectorias de movilidad. Por otro lado, está la inmersión como una práctica de aislamiento cultural de la propia cultura nacional con vistas a embeberse en la otredad cotidiana. Finalmente, la comprensión del espacio de la universidad como un campus internacional, un simulacro de atlas que concentra a estudiantes de diversas partes del mundo.

La interculturalidad es promocionada como uno de los grandes atributos de la MAI (ver por ejemplo BALDASSAR \& MCKENZIE, 2016 Y FORSEY et al., 2012) y resultaría de una idea integrativa de globalización en la cual los y las estudiantes vendrían a desarrollar una ciudadanía global (HIGGINS; BRADY, 2016; WYNVEEN; KYLE; TARRANT, 2012). El concepto de interculturalidad se basa en la experiencia en otro país como promotora de competencias culturales (FORSEY; BROOMHALL; DAVIS, 2012; HARRISON, 2015) y prácticas de desarrollo de habilidades personales de tolerancia, reflexividad y convivencia. Argumento que esa es una práctica normativa en la medida en que sitúa la cultura en una escala nacional, ignorando que las relaciones espaciales son complejas, diversas e inestables. Ese entendimiento normativo acerca de lo cultural direcciona a una comprensión de la inmersión como una estrategia por la cual opera esa interculturalidad. Los y las estudiantes se privarían de contactar a sus compatriotas y por ende intensificarían los contactos con los extranjeros (DOYLE et al., 2010; KILLICK, 2011). La manera como están planteados los dos conceptos sugiere una perspectiva despolitizada de lo multicultural, asumiendo los encuentros entre diferentes como naturales y buscando la harmonía/neutralidad, relegando relaciones de poder construidas históricamente a partir de los discursos sobre los cuerpos (conforme crítica de YOUDELL, 2006).

Moduladas en esa generalización entre nación y sujeto, las políticas de internacionalización, al promocionar ideas de la diferencia en juegos de poder entre lo local y lo global, promueven una idea de campus internacional. Lo internacional sería el alcance a los distintos espacios del globo y los estudiantes extranjeros los medios de promoverlo. Van Oorshot (2013) muestra que en esos encuentros entre estudiantes extranjeros, la cultura (metaforizada en la nación) se mueve con tonos de exotismo y curiosidad entre los grupos. Esa cartografía de estereotipos es vehículo de discursos lineales entre nación, raza, clase y cultura, como si se pudiera resumir el mundo a una sala de clases. Ese planteamiento produce una idea de cultura exótica y de la otredad espacial como una distribución territorial homogénea, lo que hace que los estudiantes vivan la frontera como un afecto geopolítico en sus cuerpos.

\section{Algunos Descontinuos EN LOS ESTUDIOS DE MOVILIDAD: APORTES POST CRÍTICOS}

Hablo de escala y cartografías por entender que estas no son solamente prácticas representativas, sino dominios epistémicos que reinstalan diferencias entre los espacios y que son vividos por los estudiantes como geopolíticas encarnadas. De esa manera, hago una distinción entre movimiento y flujo, pues este último está establecido bajo una grilla geográfica fija, dado que asume la movilidad como un comienzo y un fin, ambos análogos a orígenes y destinaciones espaciales. Los movimientos, por su parte, no son precisamente actos de comando, sino navegaciones en situaciones donde ya nos encontramos, o sea, un espacio de manobra de constante imprevisibilidad (MASSUMI, 2015).

Por ello, para poder posicionarme como investigador en movilidad y hacer confabulaciones acerca de cómo las escalas tensionan distintas prácticas de conocer, es necesario situarme en un entremedio de intensidades y moverme escapando de las definiciones que me reducen (MARTINEZ, 2018b). Ni sólo brasileño, ni sólo chileno. Ni extranjero, ni nacional. Ni objeto, ni sujeto. Ni profesor, ni estudiante. Mi cuerpo se entiende por el conjunto visceral en simultaneidad con los espacios en que vivo, siendo el afecto lo que busco para tener la posibilidad de entender las fuerzas de los encuentros que encarno y que quiero estudiar. Por lo tanto, promuevo una posición de ambigüedad escalar (BARAD, 2001).

El afecto es un concepto importante para la comprensión de que los cuerpos no son fronteras de sí mismos, sino que vienen siendo tras las intensidades políticas que se conforman entre otros cuerpos, humanos o no humanos. Por consiguiente, no somos efecto de las intensidades geopolíticas que vivemos, sino que nos vamos modulando en/con ellas. Siendo el afecto una forma compleja de conocer, entiendo la movilidad como una producción simultánea y translocal (LOW, 2016) de conocimientos escalares. Yo tomo en cuenta esa noción de afecto como circulación de poder porque las geografías actúan afectivamente, repitiendo señales, gatillando las entradas y sintonizando a los cuerpos mientras activan sus capacidades de manera diferenciada (parafraseando a MASSUMI, 2015, p.56). En ese sentido, los cuerpos son entendidos por su incertidumbre y la movilidad por su fuerza disruptiva.

Finalmente, resalto pensar la movilidad académica internacional a partir de la idea de evento, definido como el "conjunto de sorpresas - brechas, fisuras, rupturas - que ocurren performativamente para que el cuerpo busque otras maneras de resolución de los problemas" (KAISER, 2012, P. 1048, traducción propia). Por lo 
tanto, la performatividad estaría como una herramienta importante para desnaturalizar la producción social del espacio, ya que conforme señalan Kaiser y Nikiforova (2008), comprende el proceso en un devenir. Mientras que los eventos (KAISER, 2012), disyunciones en la linealidad de las trayectorias, abrirían lugar para una comprensión no unitaria, no previsible y encarnada de las prácticas estudiantiles.

\section{REFERENCIAS}

ABD AZIZ, Mohd Ismail; ABDULLAH, Doria. Finding the next 'wave' in internationalisation of higher education: focus on Malaysia. Asia Pacific Education Review, v. 15, n. 3, p. 493-502, 2014.

BALDASSAR, Loretta; MCKENZIE, Lara. Beyond "Just Being There": Teaching Internationalization at Home in Two Qualitative Methods Units. Teaching Sociology, v. 44, n. 2, p. 84-95, fev. 2016.

BARAD, Karen. Re (con)figuring space, time, and matter. Feminist locations: Global and local, theory and practice, v. $75109,2001$.

BARNETT, George A. et al. The flow of international students from a macro perspective: a network analysis. Compare: A Journal of Comparative and International Education, v. 7925 , p. 1-27, 2015.

BRENNER, Neil. The limits to scale? Methodological reflections on scalar structuration. Progress in human geography, v. 25, n. 4, p. 591-614, 2001.

BRODIN, Jane. Education for Global Competencies. Journal of Studies in International Education, v. 14, n. 5, p. 569-584, 2010.

BROWN, Michael; KNOPP, Larry. Places or polygons? Governmentality, scale, and the census in the Gay and Lesbian Atlas. Population, Space and Place, v. 12, n. 4, p. 223-242, 2006.

BROWN, Michael; KNOPP, Larry. Queering the Map: The Productive Tensions of Colliding Epistemologies. In: DODGE, Martin; KITCHIN, Robin; PERKINS, Chris. The Map Reader: Theories of Mapping Practice and Cartographic Representation, Reino Unidido: v. 98, p. 456-463, 2011.

CEFAI, Sarah. Introduction: cartographies of belonging: the marketisation of desire through media, practice and place. Gender, Place \& Culture, v. 21, n. 5, p. 582-588, 2014.

CHANKSELIANI, Maia. Escaping Homelands with Limited Employment and Tertiary Education Opportunities: Outbound Student Mobility from Post-Soviet Countries. Population, Space and Place, v. 22, n. 3, p. 301-316, 2016.
CHIANG, Li-Chuan. Trading on the West\&rsquo;s Strength: The Dilemmas of Transnational Higher Education in East Asia. Higher Education Policy, v. 25, n. 7, p. 171-189, 2012.

COLLINGE, Chris. The différance between society and space: Nested scales and the returns of spatial fetishism. Environment and Planning D: Society and Space, v. 23, n. 2, p. 189-206, 2005.

COLLINS, Francis. Globalising higher education in and through urban spaces: Higher education projects, international student mobilities and trans-local connections in Seoul. Asia Pacific Viewpoint, v. 55, n. 2, p. 242-257, 2014.

COLLINS, Francis et al. Mobility and desire: international students and Asian regionalism in aspirational Singapore. Discourse: Studies in the Cultural Politics of Education, v. 35, n. 5, p. 37-41, 2014.

DELEUZE, Gilles; GUATTARI, Felix. Mil Platôs: Capitalismo e Esquizofrenia. São Paulo: Editora 34, 1997.

DENNIS, Marguerite J. Consider implications of Brexit for international student mobility. Enrollment Management Report, v. 20, n. 6, p. 3, 2016.

DENZIN, Norman; GIARDINA, Michael. Qualitative Inquiry in Neoliberal Times. Estados Unidos: Taylor \& Francis, 2017.

DOERR, Neriko Musha. Desired learning, disavowed learning: Scale-making practices and subverting the hierarchy of study abroad experiences. Geoforum, v. 54, p. 70-79, 2014

DOLBY, N. Research in International Education Review of Educational Research, v. 78, n. 3, p. 676-726, 2008.

DOUGLASS, John Aubrey. International Berkeley: Past and Current Debates on the Role of International Students in an American University. Educational Studies, v. 1, n. 2, p. 76-108, 2015.

DOYLE, Stephanie et al. An Investigation of Factors Associated With Student Participation in Study Abroad. Journal of Studies in International Education, v. 14, n. 5, p. 471-490, 2010.

FARRUGIA, Christine A. Open Doors 2015: Report on International Educational Exchange. Estados Unidos: Institute of International Education, 2016.

FERNÁNDEZ, Pablo Iván Azócar; BUCHROITHNER, Manfred. Paradigms in cartography: an epistemological review of the 20th and 21st centuries. Reino Unido: Springer Science \& Business Media, 2013.

FINDLAY, Allan M. An Assessment of Supply and Demand-side Theorizations of International Student Mobility. International Migration, v. 49, n. 2, p. 162-190, 2011. 
FINDLAY, Allan M. et al. World class? An investigation of globalisation, difference and international student mobility. Findlay, 2011. In: Transactions of the Institute of British Geographers: Wiley Online Library, 2012. p. 118-131.

FORSEY, Martin; BROOMHALL, Susan; DAVIS, Jane. Broadening the mind? Australian student reflections on the experience of overseas study. Journal of Studies in International Education, v. 16, n. 2, p. 128-139, 2012.

FURUKAWA, Takao; SHIRAKAWA, Nobuyuki; OKUWADA, Kumi. An empirical study of graduate student mobility underpinning research universities. Higher Education, v. 66, n. 1, p. 17-37, 2013.

GAO, Xuesong. "Floating elites": Interpreting mainland Chinese undergraduates' graduation plans in Hong Kong. Asia Pacific Education Review, v. 15, n. 2, p. 223-235, 2014.

GEDDIE, Kate. Policy mobilities in the race for talent: Competitive state strategies in international student mobility. Transactions of the Institute of British Geographers, v. 40, n. 2, p. 235-248, 2015.

GONG, X.; HUYBERS, T. Chinese students and higher education destinations: Findings from a choice experiment. Australian Journal of Education, v. 0, p. 1-23, 2015.

HARRISON, Neil. Practice, problems and power in 'internationalisation at home': critical reflections on recent research evidence. Teaching in Higher Education, v. 20, n. 4, p. 412-430, 2015.

HEALEY, Nigel; MICHAEL, Lucy. Towards a New Framework for Analysing Transnational Education. Higher Education Policy, [S. 1.], v. 28, n. 3, p. 369-391, 2014.

HIGGINS, Robert M.; BRADY, Alan. Language policy, planning, and enactment: the necessity and empowering potential at the local level. Current Issues in Language Planning, v. 17, n. 3-4, p. 242-259, 2016.

KAISER, Robert. Reassembling the Event: Estonia's 'Bronze Night'. Environment and Planning D: Society and Space, v. 30, n. 6, p. 1046-1063, 2012.

KAISER, Robert; NIKIFOROVA, Elena. The performativity of scale: the social construction of scale effects in Narva, Estonia. Environment and Planning D: Society and Space, v. 26, n. 3, p. 537-562, 2008.

KILLICK, David. Journal of Studies in International Education Seeing-Ourselves-in-the- World: Developing Global Citizenship Through International Mobility and Campus Community. Journal of Studies in International Education, v. 20, n. 10, p. 1-18, 2011.

KING, Russell; RAGHURAM, Parvati. International Student Migration: Mapping the Field and New Research Agendas. Population, Space and Place, v. 19, n. 2, p. 127-137, 2013.
LEGG, Stephen; BROWN, Michael. Moral regulation : historical geography and scale. Journal of Historical Geography, v. 42, p. 134-139, 2013.

LO-PHILIP, Stephanie Wing Yan et al. Transforming educational practices: Cultural learning for short-term sojourners. International Journal of Intercultural Relations, v. 49, p. 223-234, 2015.

LOW, Setha. Spatializing Culture: The ethnography of space and place. Estados Unidos: Taylor \& Francis, 2016. https://doi.org/10.4324/9781315671277

LYOTARD, Jean-François. The Postmodern Condition: a report on knowledge. Minneapolis: University of Minnesota, 1984.

MARSTON, Sallie. The social construction of scale. Progress in Human Geography, v. 24, n. 2, p. 219-242, 2000.

MARSTON, Sallie; SMITH, Neil. States, scales and households: limits to scale thinking? A response to Brenner. Progress in human geography, v. 25, n. 4, p. 615-619, 2001.

MARTINEZ, César Augusto Ferrari. Por uma pedagogia do espaço. Boletim Gaúcho de Geografia, v. 39, n. 1-2, 2012.

MARTINEZ, César Augusto Ferrari. Entrevista com Erik Swyngedouw. Geosul, v. 33, n. 67, p. 277-287, 2018a.

MARTINEZ, César Augusto Ferrari. Espaços distorcidos: feminismo, teorias queer e geografias. Caderno Prudentino de Geografia, v. 1, n. 40, p. 52-67, 2018 b.

MASSEY, Doreen. Pelo espaço: uma nova política da espacialidade. Rio de Janeiro: Bertrand Brasil, 2008.

MASSUMI, Brian. Politics of affect. Estados Unidos: John Wiley \& Sons, 2015.

MATUS, Claudia. Imagining Time and Space in Universities. Basingstoke: Palgrave-McMillan, 2016.

MATUS, Claudia; TALBURT, Susan. Spatial imaginaries: universities, internationalization, and feminist geographies. Discourse: Studies in the Cultural Politics of Education, v. 30, n. 4, p. 515-527, 2009.

MATUS, Claudia; TALBURT, Susan. Producing global citizens for the future: space, discourse and curricular reform. Compare: A Journal of Comparative and International Education, v. 45, n. 2, p. 226-247, 2015.

NELSON, Lise. Bodies (and spaces) do matter: the limits of performativity. Gender, Place and Culture: A Journal of Feminist Geography, v. 6, n. 4, p. 331-353, 1999.

PERKINS, Richard; NEUMAYER, Eric. Geographies of educational mobilities: Exploring the uneven flows of international students. Geographical Journal, v. 180, n. 3, p. 246-259, 2014. 
PHERALI, T. J. Academic Mobility, Language, and Cultural Capital: The Experience of Transnational Academics in British Higher Education Institutions. Journal of Studies in International Education, v. 16, n. 4, p. 313-333, 2012.

RIZVI, Fazal. Towards cosmopolitan learning.

Discourse: Studies in the Cultural Politics of Education, v. 30, n. 3, p. 253-268, 2009.

RIZVI, Fazal. Theorizing student mobility in an era of globalization. Teachers and Teaching: theory and practice, v. 17, n. 6, p. 693-701, 2011.

SCHARTNER, Alina. The effect of study abroad on intercultural competence: A longitudinal case study of international postgraduate students at a British university. Journal of Multilingual and Multicultural Development, v. 37, n. 4, p. 402-418, 2016.

SHIELDS, Robin. Globalization and International Student Mobility: A Network Analysis. Comparative and Internatioanl Education Society, v. 57, n. 4, p. 609-636, 2014a.

SHIELDS, Robin. Reconsidering regionalisation in global higher education: student mobility spaces of the European Higher Education Area. Compare: A Journal of Comparative and International Education, v. 7925, p. 1-19, dec. 2014b.

SIBOMANA, Emmanuel. "We Know What To Say, We Know What To Write, But We Don'T Know How": the Challenges of Becoming Academically Literate in a New Linguistic and Socio-Cultural Space. Education as Change, v. 20, n. 2, p. 123-144, 2016.

SIDHU, Ravinder; DALL'ALBA, Gloria. International education and (dis)embodied cosmopolitanisms. Educational philosophy and theory, v. 44, n. 4, p. 413-431, 2012.

SMITH, Neil. Geography, difference and the politics of scale. In: Postmodernism and the social sciences. Estados Unidos: Springer, 1992. p. 57-79.

STERZUK, Andrea. 'The standard remains the same': language standardisation, race and othering in higher education. Journal of Multilingual and Multicultural Development, v. 4632, n. March, p. 1-14, 2014.

SWYNGEDOUW, Erik. Globalisation or "Glocalisation"? Networks, Territories and Re-Scaling. Cambridge Review of International Affairs, v. 17, n. 1, p. 1-46, 2004.

TANNOCK, Stuart. When the demand for educational equality stops at the border: wealthy students, international students and the restructuring of higher education in the UK. Journal of Education Policy, v. 28, n. 4, p. 449-464, 2013.

UNESCO. Global Flow of Tertiary-level students.

Disponível em: http://www.uis.unesco.org/Education/ Pages/international-student-flow-viz.aspx. Acesso em: $9 \mathrm{dez}$. 2016.
VAN OORSCHOT, Irene. Negotiating knowledges abroad: non-Western students and the global mobility of knowledge. Compare: A Journal of Comparative and International Education, v. 44, n. 6, p. 895-915, 2014.

WALKER, P. International Student Policies in UK Higher Education from Colonialism to the Coalition: Developments and Consequences. Journal of Studies in International Education, v. 18, n. 4, p. 325-344, 2013.

WATERS, Johanna L.; LEUNG, Maggi W. H. Domesticating transnational education: discourses of social value, self-worth and the institutionalisation of failure in 'meritocratic' Hong Kong. Transactions of the Institute of British Geographers, v. 42 , n. 2, p. 233-245, 2016.

WEISS, Meredith L.; FORD, Michele. Temporary Transnationals: Southeast Asian Students in Australia. Journal of Contemporary Asia, v. 41, n. 2 p. 229-248, 2011.

WYNVEEN, Christopher J.; KYLE, Gerard T.; TARRANT, Michael A. Study Abroad Experiences and Global Citizenship: Fostering Proenvironmental Behavior. Journal of Studies in International Education, v. 16, n. 4, p. 334-352, 2012.

YE, Rebecca. Transnational Higher Education Strategies into and out of Singapore: Commodification and Consecration. TRaNS: Trans -Regional and -National Studies of Southeast Asia, v. 4, n. 1, p. 85-108, 2015.

YOUDELL, Deborah. Subjectivation and performative politics - Butler thinking Althusser and Foucault: intelligibility, agency and the raced-nationed-religioned subjects of education. British Journal of Sociology of Education, v. 27, n. 4, p. 511-528, 2006.

ZIGURAS, Christopher; PHAM, Anh Thi Ngoc. Assessing participation in cross-border higher education in cities: Foreign education provision in Ho Chi Minh City. Asia Pacific Viewpoint, v. 55, n. 2, p. 169-181, 2014.

Recebido em: 18/3/2018.

Aprovado em: 27/12/2018

Publicado em: 6/5/2019.

Endereço para correspondência:

César Augusto Ferrari Martinez

Laboratório de Estudos Geográficos e Ambientais

Instituto de Ciências Humanas - Universidade Federal de Pelotas

Rua Coronel Alberto Rosa, 154

Pelotas, RS, Brasil

Autor:

CÉsAr Augusto Ferrari MARTINEZ

Licenciado e Mestre em Geografia (UFRGS), Doutorando em Educação pela Pontificia Universidad Católica de Chile. Professor Assistente na Universidade Federal de Pelotas.

Orcid: http://orcid.org/0000-0003-1012-8975

E-mail: cesarfmartinez@yahoo.com.br 